\title{
Cubical coloring - fractional covering by cuts and semidefinite programming $\|^{*}$
}

\author{
Robert Šámal \\ Computer Science Institute, Charles University in Prague
}

received $31^{\text {st }}$ July 2012, accepted $18^{\text {th }}$ Aug. 2015.

We introduce a new graph parameter that measures fractional covering of a graph by cuts. Besides being interesting in its own right, it is useful for study of homomorphisms and tension-continuous mappings. We study the relations with chromatic number, bipartite density, and other graph parameters.

We find the value of our parameter for a family of graphs based on hypercubes. These graphs play for our parameter the role that cliques play for the chromatic number and Kneser graphs for the fractional chromatic number. The fact that the defined parameter attains on these graphs the correct value suggests that our definition is a natural one. In the proof we use the eigenvalue bound for maximum cut and a recent result of Engström, Färnqvist, Jonsson, and Thapper [An approximability-related parameter on graphs - properties and applications, DMTCS vol. 17:1, 2015, 33-66].

We also provide a polynomial time approximation algorithm based on semidefinite programming and in particular on vector chromatic number (defined by Karger, Motwani and Sudan [Approximate graph coloring by semidefinite programming, J. ACM 45 (1998), no. 2, 246-265]).

Keywords: graph coloring, maxcut, covering, cut-continuous maps

\section{Introduction}

All graphs we consider are undirected and loopless; to avoid trivialities we do not consider edgeless graphs. For a set $W \subseteq V(G)$ we let $\delta(W)$ denote the set of edges leaving $W$ and we call any set of form $\delta(W)$ a cut. Other terminology we shall be using is standard, and can be found in, e.g., [7].

Let us call a (cut) $n / k$-cover of $G$ an $n$-tuple $\left(X_{1}, \ldots, X_{n}\right)$ of cuts in $G$ such that every edge of $G$ is covered by at least $k$ of them. We define two closely related parameters of $G$. We let

$$
x(G)=\inf \left\{\frac{n}{k} \mid \text { exists } n / k \text {-cover of } G\right\}
$$

\footnotetext{
*Preliminary version of this research appeared as extended abstract [29].

$\dagger$ Email: samal@iuuk.mff. cuni.cz. Partially supported by grant GA ČR P202-12-G061. Partially supported by grant LL1201 ERC CZ of the Czech Ministry of Education, Youth and Sports.
}

1365-8050 (c) 2015 Discrete Mathematics and Theoretical Computer Science (DMTCS), Nancy, France 
and call $x(G)$ the fractional cut-covering number of $G$. Its rescaling

$$
\chi_{q}(G)=\frac{2}{2-x(G)}
$$

will be called the cubical chromatic number of $G$. This terminology is motivated by analogy with the fractional and circular chromatic number, see the discussion following Equation (11) below. The rescaling function $2 /(2-x)$ serves the purpose of aligning the value with other variants of chromatic number, namely of attaining the right value for complete graphs. However, the rescaling function is far from arbitrary, as the values for other graphs are also modified in a proper way, see Theorem 5.2

If $k=1$, i.e., if we want to cover every edge at least once, then we need at least $\left\lceil\log _{2} \chi(G)\right\rceil$ of them [6]. Here we consider a fractional version. In this context we may find it surprising that $x(G)<2$ for every $G$ (Corollary 2.3.

From another perspective, $x(G)$ is the fractional chromatic number of a certain hypergraph: it has $E(G)$ as points and odd cycles of $G$ as hyperedges. This suggests that $x(G)$ is a solution of a linear program, see Equations (2) and (3) below.

The parameter $x(G)$ has found surprising use in theoretical computer science. Färnqvist, Jonsson, and Thapper [14] study the approximability of MAXCUT and its generalizations (so-called MAX- $H$ COLORING) using a suitably defined metric space. The function used to define the metric is in [9] recognized as a natural generalization of fractional covering by cuts. See the concluding remarks for further discussion.

As another point of view we note that $x(G)$ is a certain type of chromatic number, but instead of complete graphs (or Kneser graphs or circulants) which are used to define chromatic number (or fractional or circular chromatic number) it uses another graph scale. Let $Q_{n / k}$ denote a graph with $\{0,1\}^{n}$ as the set of vertices, where $x y$ forms an edge iff $d(x, y) \geq k$ (here $d(x, y)$ is the Hamming distance of $x$ and $y$ ).

Observation 1.1 A graph has $n / k$-cover if and only if it is homomorphic to $Q_{n / k}$.

Proof: If $\left(X_{1}, \ldots, X_{n}\right)$ is a cut $n / k$-cover of a graph $G$ then we can define homomorphism $f: V(G) \rightarrow$ $V\left(Q_{n / k}\right)$ as follows: for each $i$ we write $X_{i}$ as $\delta\left(W_{i}\right)$; we put $f(v)=1$ if $v \in W_{i}$ and $f(v)=0$ otherwise. Now $f=\left(f_{1}, \ldots, f_{n}\right)$ is a homomorphism. If, on the other hand, we are given a homomorphism $f$ : $V(G) \rightarrow V\left(Q_{n / k}\right)$ then we can put $W_{i}=\left\{v \in V(G): f_{i}(v)=1\right\}$ and observe that $\left(\delta\left(W_{1}\right), \ldots, \delta\left(W_{n}\right)\right)$ is a cut $n / k$-cover.

The above observation implies that an alternative definition of $x(G)$ is

$$
x(G)=\inf \left\{\frac{n}{k} \mid G \stackrel{\text { hom }}{\longrightarrow} Q_{n / k}\right\} .
$$

This is analogous to the definition of fractional chromatic number by means of homomorphisms to Kneser graphs (or of circular chromatic number by circulants). An immediate corollary is that $x(G)$ is a homomorphism invariant, that is if $G \stackrel{h o m}{\longrightarrow} H$ then $x(G) \leq x(H)$. This will be strengthened in Lemma 1.2 This observation suggests a possible use of the cubical chromatic number to study the structure of graph homomorphisms-we can prove nonexistence of a homomorphism $G \stackrel{\text { hom }}{\longrightarrow} H$ by showing that $x(G)>x(H)$. 
For a graph $H$ let $H^{z_{k}}$ denote the graph with vertices $V(H)$ and edges $u v$ for any $u, v \in V(H)$ with distance in $H$ at least $k$. Further let $Q_{n}$ denote the $n$-dimensional cube. Then $Q_{n / k}=Q_{n}^{\geq k}$. This corresponds to the definition of circular chromatic number, where the target graph is $C_{n}^{\geq k}$. This observation inspires the term cubical chromatic number. However, as we will see below (in Corollary 2.3, a rescaling of $x(G)$ is in order to make it behave like a version of chromatic number, thus the definition of $\chi_{q}(G)$.

The original motivation for defining $x(G)$ was the study [30, 27] of cut-continuous mappings (defined in [6]). Given graphs $G, H$ we call a mapping $f: E(G) \rightarrow E(H)$ cut-continuous, if for every cut $U \subseteq E(H)$, the preimage $f^{-1}(U)$ is a cut in $G$. The following lemma is straightforward, but useful.

Lemma 1.2 Let $G, H$ be graphs. Then if there is a cut-continuous mapping from $G$ to $H$ (in particular, if there is a homomorphism $G \stackrel{\text { hom }}{\longrightarrow} H$ ), then $x(G) \leq x(H)$ and (equivalently) $\chi_{q}(G) \leq \chi_{q}(H)$.

Proof: It suffices to show that whenever $H$ has an $n / k$-cover, $G$ has it as well. So let $f$ be some cutcontinuous mapping from $G$ to $H$, let $X_{1}, \ldots, X_{n}$ be an $n / k$-cover and consider $X_{i}^{\prime}$-a preimage of the cut $X_{i}$ under $f$. By definition, $X_{i}^{\prime}$ is also a cut. If $e$ is an edge of $G, f(e)$ is an edge of $H$, hence it is covered by at least $k$ of the cuts $X_{i}$. Thus $e$ is covered by at least $k$ of the cuts $X_{i}^{\prime}$. For the homomorphism part, one may observe that the mapping induced on edges by a homomorphism is cut-continuous [6], or just use the alternative definition in Equation (11).

As each graph $Q_{n / k}$ is a Cayley graph on $\mathbb{Z}_{2}^{n}$, it follows [30] that for every graph $G$ the existence of a homomorphism from $G$ to $Q_{n / k}$ is equivalent to the existence of a cut-continuous mapping from $G$ to $Q_{n / k}$. Consequently, we may as well use cut-continuous mapping to $Q_{n / k}$ in Equation (1). This also provides an indirect proof of Lemma 1.2 .

It is a standard exercise to show that $x(G)$ is the solution of the following linear program $(\mathcal{C}$ denotes the family of all cuts in $G$ )

$$
\text { minimize } \sum_{X \in \mathcal{C}} w(X) \text { subject to: for every edge } e, \sum_{X, e \in X \in \mathcal{C}} w(X) \geq 1 \text {. }
$$

We conclude that we can replace inf by min in the definition of $x(G)$ - the infimum is always attained. We can also consider the dual program

$$
\text { maximize } \sum_{e \in E(G)} y(e) \text { subject to: for every cut } X, \sum_{e, e \in X} y(e) \leq 1 .
$$

This program is useful for computation of $x(G)$ for some $G$. (Färnqvist, Jonsson, and Thapper [14] used a modification of this program. There is an optimal solution $y^{*}$ of the above program, that respects symmetries of $G$ : if there is an automorphism of $G$ that maps edge $e$ to edge $f$, then $y^{*}(e)=y^{*}(f)$. This decreases the size of the linear program for graphs with nontrivial automorphism group.) Moreover, in the final section we use this dual program to discuss yet another definition of $x(G)$ in terms of the bipartite subgraph polytope.

There is another possibility to dualize the notion of fractional cut covering, namely fractional cycle covering. Bermond, Jackson and Jaeger [2] proved that every bridgeless graph has a cycle $7 / 4$-cover (i.e., a collection of 7 cycles, that cover every edge at least 4 times), and Fan [11] proved that it has a 10/6-cover. 
An equivalent formulation of the Berge-Fulkerson conjecture claims that every cubic bridgeless graph has a 6/4-cover. On the other hand, Edmonds' characterization [8] of the matching polytope implies that every cubic bridgeless graph has a cycle $3 k / 2 k$-cover (for some $k$ ). It is open, whether for some fixed $k$ every cubic bridgeless graph has a cycle $3 k / 2 k$-cover.

\section{Basic properties}

In this section we discuss how the cubical chromatic number relates to other graph parameters and prove analogs of some basic results about chromatic number.

We let $\operatorname{MAXCUT}(G)$ denote the number of edges in the largest cut in $G$ and write

$$
b(G)=\operatorname{MAXCUT}(G) /|E(G)|
$$

for the bipartite density of $G$.

Lemma 2.1 For any graph $G$ it holds $x(G) \geq 1 / b(G)$. If $G$ is edge-transitive, then equality holds.

Proof: Suppose $x(G)=n / k$ and let $X_{1}, \ldots, X_{n}$ be an $n / k$-cover. Then $\sum_{i=1}^{n}\left|X_{i}\right| \leq n \cdot b(G)|E(G)|$, on the other hand this sum is at least $k \cdot|E(G)|$, as every edge is counted at least $k$ times. This proves the first part of the lemma. To prove the second part, let $\mathcal{X}=\left\{X_{1}, \ldots, X_{n}\right\}$ be all cuts of the maximal size (i.e., $\left|X_{i}\right|=b(G)|E(G)|$ ). From the edge-transitivity follows that every edge is covered by the same number (say $k$ ) of elements of $\mathcal{X}$. Now $k \cdot|E(G)|=\sum_{i=1}^{n}\left|X_{i}\right|=n \cdot b(G)|E(G)|$, which finishes the proof.

Corollary 2.2 Let Pt denote the Petersen graph.

$$
\begin{array}{ll}
x\left(K_{2 n}\right)=x\left(K_{2 n-1}\right)=2-1 / n & \chi_{q}\left(K_{2 n}\right)=\chi_{q}\left(K_{2 n-1}\right)=2 n \\
x\left(C_{2 k+1}\right)=1+1 /(2 k) & \chi_{q}\left(C_{2 k+1}\right)=2+2 /(2 k-1) \\
x(\mathrm{Pt})=5 / 4 & \chi_{q}(\mathrm{Pt})=8 / 3
\end{array}
$$

In the following result, $g_{o}(G)$ denotes the odd girth, that is, the length of a shortest odd cycle in $G$.

Corollary 2.3 For any graph $G$,

$$
2+\frac{2}{g_{o}(G)-2} \leq \chi_{q}(G) \leq 2\left\lceil\frac{\chi(G)}{2}\right\rceil .
$$

Equivalently, $1+\frac{1}{g_{o}(G)-1} \leq x(G) \leq 2-\frac{1}{\lceil\chi(G) / 2\rceil}$.

In particular, $x(G) \in[1,2)$ and $\chi_{q}(G) \geq 2$.

Proof: Let $l=g_{o}(G)$, i.e., $C_{l}$ is the shortest odd cycle that is a subgraph of $G$. Put $n=\chi(G)$. Then there are homomorphisms $C_{l} \rightarrow G \rightarrow K_{n}$, so it remains to use Lemma 1.2 and Corollary 2.2

By combining Lemma 1.2 and Corollary 2.2 we get that there is no cut-continuous mapping from $K_{n+2}$ to $K_{n}$. As there is obviously a cut-continuous mapping (indeed, even a homomorphism) in the other direction, we conclude that the even cliques $K_{2 n}$ form a strictly ascending chain in the poset defined 
by cut-continuous mappings. This application was the original point in defining $x(G)$. The result is not as straightforward as it appears (for example, there is a cut-continuous mapping $K_{4} \rightarrow K_{3}$ ).

Next, we will study how good are the bounds of Corollary 2.3 While they obviously are tight for $G$ equal to a complete graph, resp. odd cycle, they can be arbitrarily far off, as documented by Corollary 2.5 and Theorem 2.6. Before we get to that we need to look at $\chi_{f}(G)$-the fractional chromatic number of $G$. This may be defined by $\chi_{f}(G)=\inf \{n / k \mid G \stackrel{h o m}{\longrightarrow} K(n, k)\}$, where $K(n, k)$ is the Kneser graph.

Lemma 2.4 Let $k, n$ be integers such that $0<2 k \leq n$. Then

1. $b(K(n, k)) \geq 2 k / n$.

2. $x(K(n, k)) \leq n /(2 k)$.

Consequently, for any graph $G$ we have $x(G) \leq \frac{1}{2} \chi_{f}(G)$.

(Note that the bound is only useful if $k>n / 4$. Also note that the bound in part 1 . is not optimal in general; the exact value of $b(K(n, k))$ is open [3].)

Proof: For the first part we let $U=\{S \subseteq[n] \mid 1 \in S\}$ and observe that $\delta(U)$ contains $\left(\begin{array}{c}n-1 \\ k-1\end{array}\right)\left(\begin{array}{c}n-k \\ k\end{array}\right)$ edges. As Kneser graphs are edge-transitive, the second part follows by Lemma 2.1. The rest follows by Lemma 1.2 and the definition of fractional chromatic number.

Corollary 2.5 For every $\varepsilon>0$ and every integer $b$ there is a graph $G$ such that

$$
\chi_{q}(G)<2+\varepsilon \quad \text { and } \quad \chi(G)>b .
$$

Proof: Let $G=K(n, k)$, for $n=2 k+t, k=t^{2}$ and $t$ large enough. Then by Corollary 2.4 we have $x(G) \leq n / 2 k=1+t /\left(2 t^{2}\right)$, thus (for $t$ large enough) $\chi_{q}(G) \leq 2+\varepsilon$. On the other hand, it is known [25] that $\chi(G)=n-2 k+2=t+2$. Cf. also Corollary 5.4 below, where a stronger result is proved using semidefinite approximation.

By Corollary 2.3, we can view Corollary 2.5 as a strengthening of the well-known fact [10] that there are graphs with no short odd cycle and with a large chromatic number. It also shows that the converse of Lemma 1.2 is far from being true: just take $G$ from the Corollary 2.5 and let $H=K_{b / 2}$ (for $b$ large). Then $\chi_{q}(G)$ is close to 2 and $\chi_{q}(H)$ is at least $b / 2$, still by an application of Proposition 6.7 of [6] there is no cut-continuous mapping from $G$ to $H$.

It is interesting to find how various graph properties affect $\chi_{q}(G)$. From the values in Corollary 2.2 we might think that $\chi_{q}(G)$ is always larger than the fractional chromatic number $\chi_{f}(G)$. However, this is very far from the truth, as shown in Corollary 5.4 below. We saw already that small $\chi(G)$ makes $\chi_{q}(G)$ small (Corollary 2.3), while large $\chi(G)$ does not force $\chi_{q}$ to be large (Corollary 2.5). Also small $g_{o}(G)$ makes $\chi_{q}(G)$ large (Corollary 2.3 again). Complementing Corollary 2.5 we show that large $g_{o}(G)$ does not make $\chi_{q}(G)$ small (but cf. Question 2.7.

Theorem 2.6 For any integers $k, l$ there is a graph $G$ such that $\chi_{q}(G)>k$ and $G$ contains no circuit of length at most $l$. 
Proof: We modify the famous Erdős' proof of existence of high-girth graphs of high chromatic number.

Let $p=n^{\alpha-1}$ (where $\alpha \in(0,1 / l)$ ) and consider the random graph $G(n, p)$. The expected number of circuits of length at most $l$ is $O\left((p n)^{l}\right)=o(n)$, therefore by Markov inequality with probability $1-o(1)$ the graph $G(n, p)$ contains at most $n$ circuits of length at most $l$.

Using Lemma 3.1. and in particular its Claim 1, where we put $\delta=n^{-\alpha / 3}$ we get that a.a.s. $b(G(n, p)) \leq$ $\frac{1}{2}\left(1+O\left(n^{-\alpha / 3}\right)\right)$ and $|E(G(n, p))|>n^{1+\alpha} / 3$.

We take a graph $G^{\prime}$ satisfying all these three requirements. Then we delete one edge from each of the at most $n$ short circuits and let $G$ be the resulting graph.

Clearly $G$ contains no short cycles. To show $\chi_{q}(G)$ is large it is enough to show that $x(G)$ can be arbitrary close to 2 , or (using Lemma 2.1) to show that $b(G)$ can be arbitrary close to $1 / 2$.

As $\left|E\left(G^{\prime}\right)\right|=\Omega\left(n^{1+\alpha}\right)$, and as we delete at most $n$ edges of $G^{\prime}$ to get $G$, we have $|E(G)| \geq$ $\left|E\left(G^{\prime}\right)\right|(1-o(1))$. Obviously, MAXCUT in $G$ cannot be larger than in $G^{\prime}$, thus $b(G) \leq b\left(G^{\prime}\right)(1+o(1))=$ $\frac{1}{2}(1+o(1))$, which finishes the proof.

In the previous result it was crucial that the graphs had large degrees. For graphs of small degree the situation differs:

Question 2.7 Let $G$ be a cubic graph with no cycle of length $\leq c$. How large can $\chi_{q}(G)($ resp. $x(G))$ be?

For $c=3$, it follows from Brooks' theorem that $x(G) \leq x\left(K_{3}\right)=3 / 2\left(\chi_{q}(G) \leq 4\right)$. For $c=17$, it is known [5] that $G$ has a cut-continuous mapping to $C_{5}$, hence $x(G) \leq x\left(C_{5}\right)=5 / 4\left(\chi_{q}(G) \leq 8 / 3\right)$. Kardoš, Král' and Volec [22] prove that if the girth of a cubic graph $G$ is large enough, then $x(G) \leq$ $1.127752\left(\chi_{q}(G) \leq 2.2929258651\right)$. On the other hand, there is $\varepsilon>0$ such that cubic graphs $G$ of arbitrary high girth exist with $b(G)<1-\varepsilon$ (an unpublished result of McKay, see also [30]), hence with $x(G)>1+\varepsilon$ and so $\chi_{q}(G)>2+2 \varepsilon$.

We conclude this section by a simple lemma that shows that $\chi_{q}$ and $x$ enjoy some of the properties of other chromatic numbers. (Here $G_{1} \square G_{2}$ denotes the Cartesian product of graphs, $G_{1} \times G_{2}$ the categorical one (also called tensor product); for more information about graph products we refer to Imrich and Klavžar [19].)

Lemma 2.8 1. $x(G)=\max \left\{x\left(G^{\prime}\right) \mid G^{\prime}\right.$ is a component of $\left.G\right\}$

2. $x(G)=\max \left\{x\left(G^{\prime}\right) \mid G^{\prime}\right.$ is a 2-connected block of $\left.G\right\}$ for a connected graph $G$.

3. $x\left(G_{1} \square G_{2}\right)=\max \left\{x\left(G_{1}\right), x\left(G_{2}\right)\right\}$

4. $x\left(G_{1} \times G_{2}\right) \leq \min \left\{x\left(G_{1}\right), x\left(G_{2}\right)\right\}$

The same formulas are true for $\chi_{q}$ in place of $x$.

Proof: We will prove that if $G^{\prime}, G^{\prime \prime}$ are graphs that share at most one vertex, then $x\left(G^{\prime} \cup G^{\prime \prime}\right)=$ $\max \left\{x\left(G^{\prime}\right), x\left(G^{\prime \prime}\right)\right\}$. Clearly, this proves 1 and 2. Let $x\left(G^{\prime}\right)=n / k$, and $x\left(G^{\prime \prime}\right)=m / l$ (by discussion after Equation (2) the infimum is attained) and suppose $X_{1}^{\prime}, \ldots, X_{n}^{\prime}$ is an $n / k$-cover of $G^{\prime}$, while $X_{1}^{\prime \prime}, \ldots$, $X_{m}^{\prime \prime}$ is an $m / l$-cover of $G^{\prime \prime}$. Consider the collection of $m n$ cuts $\left\{X_{i}^{\prime} \cup X_{j}^{\prime \prime}\right\}$ (these are cuts, indeed, as $G^{\prime}$ and $G^{\prime \prime}$ share at most one vertex). An edge of $G^{\prime}$ is covered at least $m k$ times, an edge of $G^{\prime \prime}$ at least $n l$ times. Hence $x(G) \leq \frac{m n}{\min \{m k, n l\}}=\max \left\{\frac{n}{k}, \frac{m}{l}\right\}=\max \left\{x\left(G^{\prime}\right), x\left(G^{\prime \prime}\right)\right\}$. On the other hand, both $G^{\prime}$ and $G^{\prime \prime}$ are subgraphs of $G$, hence by Lemma 1.2 the other inequality follows. 
Part 3 follows from Lemma 1.2 , as between $G_{1} \square G_{2}$ and $G_{1} \cup G_{2}$ exists a cut-continuous mapping in both directions.

Part 4 follows from Lemma 1.2 as there are homomorphisms (and therefore TT mappings) $G_{1} \times G_{2} \rightarrow$ $G_{i}$ (for $\left.i=1,2\right)$.

As $\chi_{q}=2 /(2-x)$ (which is an increasing function for the values that $x$ can attain), the results for $\chi_{q}$ follow immediately.

\section{Cubical chromatic number of random graphs}

In this section we consider the value of cubical chromatic number of random graphs. After a short technical lemma (that is also used in the proof of Theorem 2.6 we bound $\chi_{q}$ of a random graph $G(n, 1 / 2)$ using a simple self-contained proof. We complement this by a result that provides the correct order of magnitude using results from Section 5

Lemma 3.1 Let $p$, $\delta$ be functions of $n$ such that $p, \delta \in[0,1]$ and $\delta^{2} p \geq 7 \log n / n$. Then $b(G(n, p)) \leq$ $\frac{1}{2}(1+O(1 / n)+O(\delta))$ a.a.s. In particular, we have

$$
b(G(n, p)) \leq \frac{1}{2}+O\left(\sqrt{\frac{\log n}{p n}}\right) \quad \text { a.a.s. }
$$

Proof: We will prove that almost all graphs have "many edges but no huge cut".

Claim 1. $|E(G(n, p))|>(1-\delta) p\left(\begin{array}{c}n \\ 2\end{array}\right)$ a.a.s.

To prove this we use Chernoff inequality (as stated in Corollary 2.3 of [20]) for random variable $X=$ $|E(G(n, p))|$. It claims $\operatorname{Pr}[|X-\mathbb{E} x| \geq \delta \mathbb{E} X] \leq 2 e^{-\frac{\delta^{2}}{3} \mathbb{E} X}$ for $\delta \leq 3 / 2$ and as $\mathbb{E} X=p\left(\begin{array}{l}n \\ 2\end{array}\right)$, Claim 1 follows.

Claim 2. $\operatorname{MAXCUT}(G(n, p))<(1+\delta) p \frac{n^{2}}{4}$ a.a.s.

For a set $A \subseteq V(G(n, p))$ we let $X_{A}$ be the random variable that counts the edges leaving $A$, and put $a=|A| \leq n / 2$. By Chernoff inequality for $X_{A}$ we easily get

$$
\operatorname{Pr}\left[X_{A} \geq(1+\delta) p n^{2} / 4\right] \leq 2 e^{-\frac{\delta^{2}}{3} p a(n-a)} \leq 2 e^{-\frac{\delta^{2} p a n}{6}} .
$$

It remains to estimate the total probability of a large cut:

$$
\operatorname{Pr}\left[(\exists A) X_{A} \geq(1+\delta) p n^{2} / 4\right] \leq \sum_{a=1}^{n / 2}\left(\begin{array}{l}
n \\
a
\end{array}\right) 2 e^{-\frac{\delta^{2} p a n}{6}} \leq 2\left(\left(1+e^{-\frac{\delta^{2} p n}{6}}\right)^{n}-1\right) .
$$

For $\delta^{2} p \geq 7 \log n / n$ the last expression tends to zero, which finishes the proof of Claim 2. The rest of the proof of the lemma is a simple calculation.

Theorem 3.2

$$
\Omega(\sqrt{n / \log n}) \leq \chi_{q}(G(n, 1 / 2)) \leq O(n / \log n) \quad \text { a.a.s. }
$$


Proof: The lower bound follows by Lemma 3.1, the upper one by an application of Corollary 2.3 and the well-known fact that $\chi(G(n, 1 / 2))=O(n / \log n)$.

The above theorem is included because the proof is short and self-contained. In the next theorem we give asymptotically tight estimate of $\chi_{q}(G(n, p))$. In that, however, we rely on known estimates of $\vartheta(G(n, p))$ and the relation between $\vartheta$ and $\chi_{q}$ that we derive in Section 5 below.

Theorem $3.3 \chi_{q}(G(n, p))=\Theta(\sqrt{p n}) \quad$ a.a.s.

Proof: The result follows directly using Theorem 5.1 and Theorem 5.2 below.

\section{Measuring the scale}

In this section we will discuss the 'invariance property' of cubical chromatic number. In analogy with $\chi\left(K_{n}\right)=n, \chi_{c}\left(C_{n}^{\geq k}\right)=n / k, \chi_{f}(K(n, k))=n / k$, and 'dimension of product of $n$ complete graphs is $n$ ' we would like to prove that $x\left(Q_{n / k}\right)=n / k$. The following lemma shows that the situation is not so simple for $x$.

Lemma 4.1 Let $1 \leq k \leq n$ be integers. Then we have $x\left(Q_{n / k}\right) \leq \frac{n}{k}$. If $k$ is odd, then $x\left(Q_{n / k}\right) \leq \frac{n+1}{k+1}$.

Proof: For the first part, it suffices to consider the identical homomorphism $Q_{n / k} \stackrel{h o m}{\longrightarrow} Q_{n / k}$. For the second part, mapping $V\left(Q_{n / k}\right) \rightarrow V\left(Q_{\frac{n+1}{k+1}}\right)$ given by $\left(x_{1}, \ldots, x_{n}\right) \mapsto\left(x_{1}, \ldots, x_{n}, x_{1}+\cdots+x_{n} \bmod 2\right)$ is a homomorphism whenever $k$ is odd.

Another complication is that by Corollary 2.3 we have $x(G)<2$ for any graph $G$. However, with this exception, the bounds in Lemma 4.1 are optimal:

Theorem 4.2 Let $k, n$ be integers such that $k \leq n \leq 2 k$. Then

1. if $k$ is even and $n<2 k$ then $x\left(Q_{n / k}\right)=\frac{n}{k}$; and

2. if $k$ is odd then $x\left(Q_{n / k}\right)=\frac{n+1}{k+1}$.

Corollary 4.3 There is no homomorphism $Q_{n / k} \rightarrow Q_{n^{\prime} / k^{\prime}}$ if $1 \leq n^{\prime} / k^{\prime}<n / k \leq 2$, and $k$ is even. There is no homomorphism $Q_{t n / t k} \rightarrow Q_{n, k}$ if $t>1$ is an integer, $t k$ is odd and $1<n / k<2$.

Proof: The first part follows directly from Theorem 4.2 and Lemma 1.2 (note that $\left(n^{\prime}+1\right) /\left(k^{\prime}+1\right) \leq$ $n^{\prime} / k^{\prime}$, so we do not care about parity of $k^{\prime}$ ). For the second part, observe first, that $Q_{n / k}$ is a subgraph of $Q_{t n / t k}$ for a positive integer $t$. It is known [15] that $Q_{t n / t k}$ is a core, thus it does not have a homomorphism to its proper subgraph.

This theorem was announced as a conjecture in the author's thesis [30], together with a part of a possible proof. The proof was finished by Engström, Färnqvist, Jonsson, and Thapper [9. Proposition 5.11], who did prove the inequality in Lemma 4.5 .

We'll use the following result (see Lemma 13.7.4 and 13.1.2 of [16]).

Lemma 4.4 Let $G$ be an $r$-regular graph with $n$ vertices, let $\lambda_{\min }$ be the smallest eigenvalue of $G$. Then $b(G) \leq \frac{1}{2}\left(1-\frac{\lambda_{\min }}{r}\right)$. 
The following lemma was proved (using a clever induction) by Engström, Färnqvist, Jonsson, and Thapper [9. Proposition 5.11], resolving thus a question from the author's thesis [30].

Lemma 4.5 Let $k, n$ be integers such that $k \leq n<2 k$ and $k$ is even, let $x$ be an integer such that $1 \leq x \leq n$. Then

$$
\sum_{o d d t}\left(\begin{array}{l}
x \\
t
\end{array}\right)\left(\begin{array}{l}
n-x \\
k-t
\end{array}\right) \leq\left(\begin{array}{l}
n-1 \\
k-1
\end{array}\right) .
$$

Proof: (of Theorem 4.2) Since Lemma 4.1 provides the upper bound, we only need to establish the lower bound. Suppose first that $k$ is even. We shall use a spanning subgraph of $Q_{n / k}=Q_{\bar{n}}^{\geq k}$, that contains only edges of length precisely $k$; we shall use $Q_{n}^{=k}$ to denote this subgraph.

By Lemma 1.2 and 2.1 we have that $x\left(Q_{n / k}\right) \geq x\left(Q_{n}^{=k}\right)=1 / b\left(Q_{n}^{=k}\right)$. By Lemma 4.4 it is enough to determine the smallest eigenvalue $\lambda_{\min }$ of $Q_{n}^{=k}$. As $Q_{n}^{=k}$ is $\left(\begin{array}{l}n \\ k\end{array}\right)$-regular, we have

$$
\frac{1}{b\left(Q_{n}^{=k}\right)} \geq \frac{2}{1-\lambda_{\min } /\left(\begin{array}{c}
n \\
k
\end{array}\right)} .
$$

It is standard (see, e.g., Problem 11.8 in [26] or the theory of Association Schemes in Chapter 30 of [31]) that the eigenvalues of $Q_{n}^{=k}$ are

$$
\sum_{t=0}^{k}(-1)^{t}\left(\begin{array}{l}
x \\
t
\end{array}\right)\left(\begin{array}{l}
n-x \\
k-t
\end{array}\right)
$$

By using Vandermonde's identity and Lemma 4.5, we get that the above sum is at least $\left(\begin{array}{l}n \\ k\end{array}\right)(1-2 k / n)$, which is equal to the sum for $x=1$. Thus the smallest eigenvalue $\lambda_{\min }$ equals $\left(\begin{array}{l}n \\ k\end{array}\right)(1-2 k / n)$, and we obtain $x\left(Q_{n / k}\right) \geq n / k$ as desired.

For odd values of $k$ we cannot use the same method, as then $Q_{n}^{=k}$ is bipartite, hence $b\left(Q_{n}^{=k}\right)=1$. However, observe that $Q_{\frac{n+1}{k+1}} \stackrel{h o m}{\longrightarrow} Q_{n / k}$, hence by Lemma 1.2 and the result for (even) $k+1$ we have

$$
x\left(Q_{n / k}\right) \geq x\left(Q_{\frac{n+1}{k+1}}\right) \geq \frac{n+1}{k+1} .
$$

Corollary 4.6 The set $\{x(G) \mid G$ is a graph $\}$ equals $\mathbb{Q} \cap[1,2]$. Consequently, the set $\left\{\chi_{q}(G) \mid G\right.$ is a graph $\}$ equals $\mathbb{Q} \cap[2, \infty)$.

\section{Semidefinite approximation}

In this section we show how to approximate $\chi_{q}$ in polynomial time up to a factor of $\pi / 2$. Key to this approximation is the vector coloring, introduced by [23] based on the Lovász' $\vartheta$ function. The concept of vertex coloring is extended by using high-dimensional unit vectors as colors, and requiring adjacent vertices to be assigned distant vectors. Precisely: given a graph $G$ and real $t<0$ consider a mapping $f: V(G) \rightarrow \mathbb{R}^{n}$ (where $\left.n=|V(G)|\right)$, so that 
- $\|f(v)\|_{2}=1$ for every vertex $v$ and

- $\langle f(u), f(v)\rangle \leq t$ for every edge $u v$.

We let $t(G)$ denote the minimum $t$ such that function $f$ with the above properties exists. The vector chromatic number of $G$ is defined as $\chi_{v}(G)=1-\frac{1}{t(G)}$.

As these conditions for $t(G)$ can be formulated as a semidefinite program, the minimum indeed exists; more importantly, $t(G)$ can be approximated with an absolute error $\varepsilon$ in time polynomial in $n$ and $\log \frac{1}{\varepsilon}$. Indeed, Karger, Motwani and Sudan [23, Lemma 3.2] prove that if a graph $G$ has $\chi_{v}(G)=k$ then it is possible to find a vector $(k+\varepsilon)$-coloring in time polynomial in $n$ and $\log 1 / \varepsilon-$ in particular, one finds approximation to $\chi_{v}$ up to an absolute error $\varepsilon$.

It is easy to see that $\chi_{v}(G) \leq \chi(G)$ - given a proper $k$-coloring, we may map all vertices of one color to one vertex of a simplex with $k$ vertices. This will lead to $t=-\frac{1}{k-1}$, and so indeed $\chi_{v}(G) \leq k$. However, the fraction $\chi(G) / \chi_{v}(G)$ can be arbitrarily large [12], in fact as large as $n / \operatorname{polylog}(n)$ (where $n=|V(G)|)$; this contrasts sharply with Theorem 5.2 .

For further properties of $\chi_{v}$ see [23] and [4]. In the latter the following is shown.

Theorem $5.1([\overline{4}]) c_{1} \sqrt{n p} \leq \chi_{v}\left(G_{n, p}\right) \leq c_{2} \sqrt{n p}$ with probability $1-o(1)$.

Now we proceed to show to connection between $\chi_{q}$ and $\chi_{v}$.

Theorem 5.2 For every graph $G$ we have

$$
\chi_{v}(G) \leq \chi_{q}(G) \leq \frac{\pi}{2} \chi_{v}(G) .
$$

Proof: We prove the lower bound first. Recall that $\chi_{q}(G)=\frac{2}{2-x(G)}$ and $x(G)=n / k$, for some $n, k$ where there is a $k$-cover of $G$ by $n$ cuts. (The fact that the infimum in the definition of $x(G)$ is attained follows from the linear-programming reformulation, see Equation (2).) Equivalently, there is a mapping $g: V(G) \rightarrow\{ \pm 1\}^{n}$ (the $i$-th coordinate encodes the $i$-th cut so that for every edge $u v$ the vectors $g(u)$ and $g(v)$ differ in $\geq k$ coordinates. Put $f(v)=g(v) / \sqrt{n}$. Obviously, each $f(v)$ is a unit vector, while for every edge $u v$ we have

$$
\langle f(u), f(v)\rangle=1-\frac{2 d_{H}(g(u), g(v))}{n} \leq 1-\frac{2 k}{n}=1-\frac{2}{x(G)} .
$$

Therefore, for this $f$ we get $t \leq 1-2 / x(G)$. Consequently,

$$
\chi_{v}(G) \leq 1-\frac{1}{t} \leq 1-\frac{x(G)}{x(G)-2}=\frac{2}{2-x(G)}=\chi_{q}(G) .
$$

For the upper bound we use probabilistic approach, motivated by the algorithm for approximating MAXCUT by Goemans and Williamson [17]. Consider a mapping $f$ as above, the scalar products are at most $t$ with $\chi_{v}(G)=1-1 / t$. For a large $N$, we choose $N$ uniformly random hyperplanes in $\mathbb{R}^{n}$ through the origin. With probability 1 none of them contains any of the points $f(v)$ for $v \in V(G)$, therefore each hyperplane defines a cut. We shell prove that with probability $1-o(1)$ this cut covering gives us the desired bound.

To this end, consider an edge $u v \in E(G)$, let $\alpha$ be the angle between the unit vectors $f(u)$ and $f(v)$. The following elementary observation (used also in [17]) is crucial for the calculation: 
A random hyperplane through origin separates $f(u)$ and $f(v)$ with probability $\frac{\alpha}{\pi}$.

For an edge $e=u v$ let $X_{e}$ be the random variable that counts how many of the $N$ hyperplanes separate the end-vertices of $e$. Obviously, $X_{e}$ follows a binomial distribution $\operatorname{Bin}(N, p)$ with $p=\frac{\alpha}{\pi}$. We have $\cos \alpha=\langle f(u), f(v)\rangle \leq t$, so $p \geq \frac{\arccos t}{\pi}$. By the Chernoff inequality we have $\operatorname{Pr}\left[X_{e}<p N-s\right]<$ $e^{-\frac{s^{2}}{2 N p}}$. Putting $s=\left\lceil N^{2 / 3}\right\rceil$ we obtain

$$
\operatorname{Pr}\left[X_{e}<p N-\left\lceil N^{2 / 3}\right\rceil\right]<e^{-\frac{N^{1 / 3}}{2 p}}=o(1)
$$

(the $o(1)$ is with respect to $N$ growing to infinity). Thus, with probability $1-\left(\begin{array}{l}n \\ 2\end{array}\right) o(1)=1-o(1)$ we have $X_{e} \geq p N-\left\lceil N^{2 / 3}\right\rceil$ for every edge $e$. So for every large enough $N$ there is a cut covering achieving this and from the definition of $x(G)$, we get that

$$
x(G) \leq \frac{N}{p N-\left\lceil N^{2 / 3}\right\rceil}=\frac{1}{p}(1+o(1)) .
$$

As we may choose arbitrarily large $N$, we get from here that $x(G) \leq \frac{1}{p}=\frac{\pi}{\arccos t}$. Now from the definition we obtain

$$
\frac{\chi_{q}(G)}{\chi_{v}(G)}=\frac{\frac{2}{2-x(G)}}{1-\frac{1}{t}} \leq \frac{\frac{2}{2-\frac{\pi}{\arccos t}}}{1-\frac{1}{t}}=\frac{t \arccos t}{(\arccos t-\pi / 2)(t-1)}
$$

Putting $t=\cos \alpha$ and $\beta=\alpha-\pi / 2$ (so that $t=-\sin \beta$ ), the last expression equals

$$
\frac{\sin \beta}{\beta} \frac{\beta+\frac{\pi}{2}}{\sin \beta+1} \leq 1 \cdot \frac{\pi}{2}
$$

(we used the elementary estimate $\frac{2}{\pi} \beta \leq \sin \beta \leq \beta$ valid for $\beta \in[0, \pi / 2]$ ).

We note that the above proof also yields bound $\chi_{q}(G) \leq 1 /\left(1-\frac{\pi}{2 \arccos \frac{1}{1-\chi_{v}(G)}}\right)$, which is, for small values of $\chi_{v}(G)$, slightly better than the above theorem.

Corollary 5.3 There is a polynomial-time algorithm that approximates $\chi_{q}(G)$ with approximation factor almost $\frac{\pi}{2}$. More precisely: to get an approximation factor at most $\frac{\pi}{2}(1+\varepsilon)$ we need an algorithm polynomial in $|V(G)|$ and $\log 1 / \varepsilon$.

Corollary 5.4 For every graph $G$ we have

$$
\chi_{q}(G) \leq \frac{\pi}{2} \chi_{f}(G) .
$$

Moreover, there is a sequence of graphs for which $\chi_{q}(G)$ is bounded, while $\chi_{f}(G)$ is unbounded.

Proof: For the first part it is enough to use Theorem 5.2, the bound $\chi_{v}(G) \leq \vartheta(\bar{G})$ (Theorem 8.2 of [23]) and the well-known bound $\vartheta(\bar{G}) \leq \chi_{f}(G)$. We use Theorem 1.2 of Feige, Langberg, and Schechtman [12]: There are infinitely many graphs $G$ that are vector 3-colorable and satisfy $\alpha(G) \leq$ $n^{0.843}$ (where $n$ is the number of vertices of $G$ ). Each such graph $G$ satisfies $\chi_{q}(G) \leq 3 \pi / 2<5$, and $\chi_{f}(G) \geq n / n^{0.843}=n^{0.157}$. 
Let us note here an exciting development related to the above mentioned result of Feige, Langberg, and Schechtman [12]. If we are given a 3-colorable graph $G$, it is still computationally hard to find a 3coloring of it. This lead Karger, Motwani, and Sudan [23] to their definition of vector chromatic number. As $\chi_{v}(G) \leq \chi(G) \leq 3$, and vector coloring is computationally tractable, we can find a vector 3-coloring of $G$ and then use various rounding techniques to find a coloring of $G$. The best result in this direction is $O\left(n^{0.19996}\right)$ colors due to Kawarabayashi and Thorup [24]. As a limit to this approach Feige, Langberg, and Schechtman [12] observe that just using the fact that $\chi_{v}(G) \leq 3$ does not prevent a graph from having chromatic number as large as $\Omega\left(n^{0.157}\right)$, thus to efficiently color 3 -colorable graph with less colors (if at all possible), a different technique is needed.

\section{Concluding Remarks}

Bipartite subgraph polytope For a bipartite subgraph $B \subseteq G$, let $c_{B}$ be the characteristic vector of $E(B)$. Bipartite subgraph polytope $P_{B}(G)$ is the convex hull of points $c_{B}$, for all bipartite graphs $B \subseteq G$. The study of this polytope was motivated by the MAXCUT problem: looking for a weighted maximum cut of $G$ simply means solving a linear program over $P_{B}(G)$. Thus, for graphs where $P_{B}(G)$ has simple description, we can have a polynomial-time algorithm for MAXCUT; this in particular happens for weakly bipartite graphs (which include planar graphs), see [18]. We apply $P_{B}$ to yield yet another definition of $x$.

Theorem 6.1 $x(G)=\max \left\{\sum_{e \in E(G)} y_{e} \mid y \cdot c \leq 1\right.$ defines a facet of $\left.P_{B}(G)\right\}$

Proof: By LP duality $x(G)$ is a solution to the program (3). This means, that we are maximizing over such $y$, that for each cut $X$ satisfy $y \cdot c_{X} \leq 1$. As the convex hull of vectors $c_{X}$ is $P_{B}$, we are maximizing the sum of coordinates of an element of the dual polytope $P_{B}^{*}$. This maximum is attained for some vertex of $P_{B}^{*}$, that is for $y$ such that $y \cdot c \leq 1$ defines a facet of $P_{B}$.

'Natural' facets of $P_{B}(G)$ are defined by $\sum_{e \in E(H)} y_{e} \leq \operatorname{MAXCUT}(H)$ for some $H \subseteq G$. (This inequality is satisfied for every graph $H$, but it doesn't always define a face of maximal dimension.) This proves the following observation (we add a direct proof, too).

Lemma 6.2 $x(G) \geq 1 /\left(\min _{H \subseteq G} b(H)\right)$

Proof: Suppose $H \subseteq G$. Then there exists a cut-continuous mapping (indeed, a homomorphism) from $H$ to $G$, which by Lemma 1.2 and 2.1 implies $1 / b(H) \leq x(G)$.

Let us return to Lemma 2.1 for a while. In general $x(G)$ and $1 / b(G)$ can be as distant as possible: Let $G$ be a disjoint union of a $K_{n}$ and $K_{N, N}$. Now $x(G)$ is close to 2 (because $G$ is homomorphically equivalent to $K_{n}$, hence $x(G)=x\left(K_{n}\right)$ ) and $b(G)$ is close to 1 (provided $N$ is sufficiently large). This motivates Lemma 6.2 which improves the original bound. A natural question is whether this improvement gives the correct size of $x$. It turns out it does not (contrary to a conjecture in the author's thesis). In [9] it is shown, that the circular clique $K_{11 / 4}$ is a counterexample.

A failed approach The proof of Theorem 4.2 could be attempted by another way: First, observe that the Kneser graph $K(n, r)$ is a subgraph of $Q_{n / 2 r}$. By Lemmas 1.2 and 2.1 we have $x\left(Q_{n / 2 r}\right) \geq$ $x(K(n, r)) \geq \frac{1}{b(K(n, r))}$. Thus, if we knew the value of $b(K(n, r))$ (and it turned out to be $2 r / n$ for the range of $r$ we are interested in), we would be done. 
In [28] it is claimed that if $2 r \leq n \leq 3 r$ then, indeed, $b(K(n, r))=2 r / n$. This would imply the conjecture for even $k$ less than $3 / 2 \cdot n$; unfortunately the proof in [28] is incomplete (as already observed by [3]). Thus, the true value of MAXCUT for Kneser graphs remains open.

Generalizations and future work As already mentioned in the introduction, the metric that is used in [14, 9] to study approximability of MAX- $H$-COLORING can be computed from a generalization of fractional covering by cuts. One only needs to consider more general edge sets in place of cuts, namely edge sets of graphs that are homomorphic to $H$. Then the cube $Q_{n / k}$ in Equation (1) is replaced by appropriately defined power of $H$. One may also use this motivation to define $H$-continuous mappings as follows. We call a subset $X \subseteq E(G)$ an $H$-cut in $G$ whenever there is a mapping $g: V(G) \rightarrow V(H)$ for which $g^{-1}(E(H))=X$. We say a mapping $f: E\left(G_{1}\right) \rightarrow E\left(G_{2}\right)$ is $H$-continuous whenever a preimage of each $H$-cut is an $H$-cut. This notion deserves further attention. Some preliminary observations are obtained in [9].

Possible use of recent techniques for approximating MAXCUT In recent years, a lot of attention has been put to various ways to approximate MAXCUT without using semidefinite programming. In particular, finding a combinatorial approximation algorithm is of interest; a nice algorithm based on random walks exists [21]. It seems, however, that this method fails to approximate $\chi_{q}$, because it is based on local properties of the given graph, and $\chi_{q}$ can be very large even for graphs that are locally trees (see Theorem 2.6. It would be interesting, though, if such techniques could be used for approximating $\chi_{q}$ for graphs of bounded degree.

Number of cuts required By definition, if $x(G)=t$ then there is a cut $n / k$-cover for some $n, k$ satisfying $t=\frac{n}{k}$. It would be nice to know how large $n$ is required. To be precise, define $n(G)$ to be the smallest $n$ as above. Then we let

$$
f(v)=\max \{n(G) \mid G \text { is a graph with } v \text { vertices }\} .
$$

This maximum clearly exists (as there are only finitely many graphs on $v$ vertices).

Question 6.3 How fast doest $f(v)$ grow? Is $f(v) \leq 2^{v}$ ? For what graph(s) is the maximum in the definition of $f(v)$ attained?

The estimate by $2^{v}$ seems natural, as there is only $2^{v-1}$ different cuts in a graph on $v$ vertices. However, one may be forced to take some cuts repeatedly.

Complexity In view of the complexity of computing other variants of chromatic number, the following conjecture is natural. Note, however, that in contrast with chromatic or fractional chromatic number, cubical chromatic number can be approximated up to a constant factor.

Conjecture 6.4 For any $s>2$ determining if an input graph $G$ satisfies $\chi_{q}(G) \leq s$ is NP-complete.

Perhaps more importantly: how well can one approximate $\chi_{q}$ in polynomial time? Can one use the techniques of [1] to find a PTAS for $\chi_{q}(G)$ - at least in the case when $G$ is dense? It is tempting to use the ellipsoid method to solve the linear program (3), where results of [1] can serve as an (approximate) separation oracle. To do this, however, we need a PTAS for weighted MAX-CUT. While some results in this direction are known [13], they are not strong enough (the issue is that some weights may be much larger than the others, which basically makes our instance not dense). 
Cubic graphs For the reader's convenience we restate here Question 2.7. For known partial results we refer the reader to Section 2 .

Question 6.5 Let $G$ be a cubic graph with no cycle of length $\leq c$. How large can $x(G)\left(\right.$ resp. $\chi_{q}(G)$ ) be?

\section{Acknowledgement}

I thank Matt DeVos and Stephan Thomassé for suggesting to use semidefinite programming to approximate the cubical chromatic number and for helpful discussion about the topic. I thank the anonymous referees for helpful comments leading to improved presentation.

\section{References}

[1] Sanjeev Arora, David Karger, and Marek Karpinski, Polynomial time approximation schemes for dense instances of NP-hard problems, Proceedings of the twenty-seventh annual ACM symposium on Theory of computing, ACM, 1995, pp. 284-293.

[2] Jean-Claude Bermond, Bill Jackson, and François Jaeger, Shortest coverings of graphs with cycles, J. Combin. Theory Ser. B 35 (1983), no. 3, 297-308.

[3] Béla Bollobás and Imre Leader, Set systems with few disjoint pairs, Combinatorica 23 (2003), no. 4, 559-570.

[4] Amin Coja-Oghlan, The Lovász Number of Random Graphs, Combinatorics, Probability and Computing 14 (2005), no. 04, 439-465.

[5] Matt DeVos and Robert Šámal, High-girth cubic graphs are homomorphic to the Clebsch graph, J. Graph Theory 66 (2011), 241-259.

[6] Matt DeVos, Jaroslav Nešetřil, and André Raspaud, On edge-maps whose inverse preserves flows and tensions, Graph Theory in Paris: Proceedings of a Conference in Memory of Claude Berge (J. A. Bondy, J. Fonlupt, J.-L. Fouquet, J.-C. Fournier, and J. L. Ramirez Alfonsin, eds.), Trends in Mathematics, Birkhäuser, 2006.

[7] Reinhard Diestel, Graph theory, Graduate Texts in Mathematics, vol. 173, Springer-Verlag, New York, 2000.

[8] Jack Edmonds, Maximum matching and a polyhedron with 0, 1-vertices, J. Res. Nat. Bur. Standards Sect. B 69B (1965), 125-130.

[9] Robert Engström, Tommy Färnqvist, Peter Jonsson, and Johan Thapper, An approximability-related parameter on graphs-properties and applications, Discr. Math. Th. Comp. Sc. 17 (2015), no. 1, $33-66$.

[10] Paul Erdős, Graph theory and probability, Canad. J. Math. 11 (1959), 34-38.

[11] Genghua Fan, Minimum cycle covers of graphs, J. Graph Theory 25 (1997), no. 3, 229-242. 
[12] Uriel Feige, Michael Langberg, and Gideon Schechtman, Graphs with tiny vector chromatic numbers and huge chromatic numbers, SIAM J. Comput. 33 (2004), no. 6, 1338-1368.

[13] Wenceslao Fernandez de la Vega and Marek Karpinski, Polynomial time approximation of dense weighted instances of MAX-CUT, Random Structures \& Algorithms 16 (2000), no. 4, 314-332.

[14] Tommy Färnqvist, Peter Jonsson, and Johan Thapper, Approximability distance in the space of $H$ colourability problems., Proceedings of the 4th International Computer Science Symposium in Russia (CSR-2009), 2009, arXiv:0802.0423.

[15] Chris Godsil, David Roberson, Brendan Rooney, Robert Šámal, and Antonios Varvitsiotis, Rigidity and Graph Theory III: Categorical Products and Unique Homomorphisms, in preparation, 2015.

[16] Chris Godsil and Gordon Royle, Algebraic graph theory, Graduate Texts in Mathematics, vol. 207, Springer-Verlag, New York, 2001.

[17] Michel X. Goemans and David P. Williamson, Improved approximation algorithms for maximum cut and satisfiability problems using semidefinite programming, J. Assoc. Comput. Mach. 42 (1995), no. 6, 1115-1145.

[18] Martin Grötschel and William R. Pulleyblank, Weakly bipartite graphs and the max-cut problem, Oper. Res. Lett. 1 (1981/82), no. 1, 23-27.

[19] Wilfried Imrich and Sandi Klavžar, Product graphs, structure and recognition, Wiley, 2000.

[20] Svante Janson, Tomasz Łuczak, and Andrzej Rucinski, Random graphs, Wiley-Interscience Series in Discrete Mathematics and Optimization, Wiley-Interscience, New York, 2000.

[21] Satyen Kale and Comandur Seshadhri, Combinatorial Approximation Algorithms for MaxCut using Random Walks, Innovations in Computer Science - ICS 2010, Tsinghua University, Beijing, China, January 7-9, 2011. Proceedings, 2011, pp. 367-388.

[22] František Kardoš, Daniel Král', and Jan Volec, Maximum edge-cuts in cubic graphs with large girth and in random cubic graphs, Random Structures \& Algorithms 41 (2012), no. 4, 506-520.

[23] David Karger, Rajeev Motwani, and Madhu Sudan, Approximate graph coloring by semidefinite programming, J. ACM 45 (1998), no. 2, 246-265.

[24] Ken-ichi Kawarabayashi and Mikkel Thorup, Coloring 3-colorable graphs with o( $\left.n^{1 / 5}\right)$ colors, 31 st International Symposium on Theoretical Aspects of Computer Science (STACS 2014), STACS 2014, March 5-8, 2014, Lyon, France, 2014, pp. 458-469.

[25] László Lovász, Kneser's conjecture, chromatic number, and homotopy, J. Combin. Theory Ser. A 25 (1978), no. 3, 319-324.

[26] László Lovász, Combinatorial problems and exercises, North-Holland Publishing Co., Amsterdam, 1979.

[27] Jaroslav Nešetřil and Robert Šámal, On tension-continuous mappings, European J. Combin. 29 (2008), no. 4, 1025-1054. 
[28] Svatopluk Poljak and Zsolt Tuza, Maximum bipartite subgraphs of Kneser graphs, Graphs Combin. 3 (1987), no. 2, 191-199.

[29] Robert Šámal, Fractional covering by cuts, Electronic Notes in Discrete Mathematics (2005), no. 22, 455-459, Proceedings of 7th International Colloquium on Graph Theory (Hyères, 2005).

[30] Robert Šámal, On XY mappings, Ph.D. thesis, Charles University, 2006.

[31] Jack H. van Lint and Richard M. Wilson, A course in combinatorics, second ed., Cambridge University Press, Cambridge, 2001. 